\title{
Reflets
}

Revue ontaroise d'intervention sociale et communautaire

\section{Une ère de changement pour la formation en réadaptation}

Jacinthe Savard, Claire-Jehanne Dubouloz et Dawn Burnett

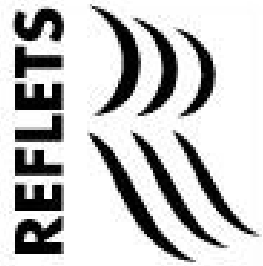

Volume 8, numéro 1, printemps 2002

La réadaptation : son visage français en Ontario

URI : https://id.erudit.org/iderudit/026375ar

DOI : https://doi.org/10.7202/026375ar

Aller au sommaire du numéro

Éditeur(s)

Reflets : Revue ontaroise d'intervention sociale et communautaire

ISSN

1203-4576 (imprimé)

1712-8498 (numérique)

Découvrir la revue

Citer cet article

Savard, J., Dubouloz, C.-J. \& Burnett, D. (2002). Une ère de changement pour la formation en réadaptation. Reflets, 8(1), 95-105.

https://doi.org/10.7202/026375ar

Tous droits réservés (C) Reflets : Revue ontaroise d'intervention sociale et communautaire, 2002
Ce document est protégé par la loi sur le droit d'auteur. L'utilisation des services d'Érudit (y compris la reproduction) est assujettie à sa politique d'utilisation que vous pouvez consulter en ligne.

https://apropos.erudit.org/fr/usagers/politique-dutilisation/ 


\section{Une ère de changement pour la formation en réadaptation}

Jacinthe Savard ${ }^{1}$

École des sciences de la réadaptation (ergothérapie), Université d'Ottawa

Claire-Jehanne Dubouloz

École des sciences de la réadaptation (ergothérapie), Université d'Ottawa

Dawn Burnett

École des sciences de la réadaptation (physiothérapie)

\section{Introduction}

L'Université d'Ottawa est la seule université ontarienne à offrir en français les programmes de formation en audiologie, en ergothérapie, en orthophonie et en physiothérapie. La mission commune de ces programmes en réadaptation est de former des professionnels bilingues capables de desservir les besoins de la population francophone de l'Ontario et d'autres communautés francophones du Canada. Les programmes d'audiologie et d'orthophonie sont offerts au niveau de la maitrise, tel que l'exigent les ordres professionnels de ces disciplines dans toutes les provinces canadiennes. Les programmes d'ergothérapie et de physiothérapie étaient jusqu'à récemment offerts au niveau du premier cycle (baccalauréat) dans toutes les universités canadiennes. Mais un vent de changement se fait sentir depuis quelques années. Bientôt, les programmes de formation de base en ergothérapie et en physiothérapie ne seront offerts eux aussi qu'au niveau de la 
maîtrise professionnelle (ACE 2001;ACP 2001). Les programmes d'ergothérapie et de physiothérapie de l'Université d'Ottawa emboîteront le pas.

Dans cet article, nous présenterons d'abord l'historique des transformations et les raisons qui ont motivé ce changement à la formation de base au sein de ces deux professions. Nous préciserons ensuite ce qu'est une maitrise professionnelle et comment nous l'envisageons à l'Université d'Ottawa. Nous discuterons de l'impact de ce changement sur notre enseignement, ainsi que sur la pratique de l'ergothérapie et de la physiothérapie. Nous terminerons avec les étapes prévues pour la transformation des programmes à l'Université d'Ottawa.

\section{Historique des transformations}

Le mouvement a commencé aux États-Unis où les programmes de baccalauréat et de maitrise professionnelle coexistaient depuis plusieurs années comme porte d'entrée à la pratique professionnelle en ergothérapie et en physiothérapie (Warren \& Pierson 1994; Polotajko 1999).En 1997,l'American Physical Therapy Association a annoncé qu'elle exigera la formation de niveau maitrise à compter de 2002 (CAPTE 1997). L'American Occupational Therapists Association ${ }^{2}$ l'exigera à compter de 2007 (AOTA 2002). Au Canada, l'Université Western (Ontario) a été la première, en 1998, à transformer son programme de baccalauréat en ergothérapie, en programme de maitrise professionnelle. À compter de septembre 2002 , trois des cinq universités ontariennes qui offrent la formation de base en ergothérapie et en physiothérapie n'offriront plus ces formations dans des programmes de baccalauréat, mais dans des programmes de maitrise professionnelle. Les associations professionnelles canadiennes ont aussi pris position. En juin 2001, les représentants de l'Association canadienne de physiothérapie (ACP), les ordres professionnels en physiothérapie de toutes les provinces canadiennes, ainsi que les directeurs de programmes 
universitaires en physiothérapie, ont émis une position commune qui consiste à travailler pour que la formation en physiothérapie ne soit offerte qu'au niveau de la maitrise professionnelle, au plus tard en 2010. En novembre 2001, l'Association canadienne d'ergothérapie (ACE) annonçait qu'à compter de 2010, elle exigerait la maitrise professionnelle comme critère d'accès pour les ergothérapeutes débutants, et à compter de 2008, comme critère d'agrément des programmes universitaires en ergothérapie.

\section{Raisons qui motivent ce passage à la maîtrise professionnelle}

Toutes les instances qui se sont prononcées pour le rehaussement du niveau de formation de base pour les futurs ergothérapeutes et physiothérapeutes invoquent les mêmes arguments. Le premier et le plus important met en évidence l'évolution de ces deux professions et des connaissances attendues des nouveaux diplômés. Comme le mentionne l'ACE : "Cette décision a été prise à la suite d'une consultation à grande échelle des membres et autres parties prenantes sur la question de la formation des ergothérapeutes. L'un des constats les plus révélateurs de cette consultation a été la reconnaissance que les compétences et connaissances exigées auparavant pour la pratique chevronnée sont maintenant essentielles dès l'entrée en pratique... " (ACE 2001 : 1). Ces nouvelles compétences exigées sont le reflet de changements dans la pratique, dont voici les principaux :

- L'augmentation de l'importance accordée à la pratique fondée sur les faits scientifiques nécessite une meilleure préparation en recherche, surtout en ce qui touche l'analyse critique des résultats de recherche (Law \& Baum 1998). Ces habiletés sont habituellement acquises au niveau de la maitrise (Polotajko 1999; Salvatori 1998; University of Alberta 2000). Une étude menée aux États-Unis, auprès de 512 finissants en physiothérapie de niveau baccalauréat et de niveau maîtrise, démontre 
que les personnes formées à la maitrise professionnelle se sentent davantage préparées pour comprendre les résultats de recherche, ainsi que pour participer elles-mêmes au développement des connaissances cliniques. De plus, par rapport à leurs collègues de niveau baccalauréat, ces finissants de niveau maitrise se sentent aussi bien préparés ou mieux préparés pour la pratique clinique (Warren \& Pierson 1994).

- La restructuration du système de santé a entrainé de nombreux changements dans la pratique clinique de l'ergothérapie et de la physiothérapie. Davantage d'ergothérapeutes et de physiothérapeutes travaillent en pratique privée ou dans des milieux communautaires où, souvent, ils ne sont pas supervisés lorsqu'ils débutent leur pratique. Avec la venue de la gestion par programme, ceux et celles qui travaillent dans des milieux plus traditionnels sont de plus en plus souvent les seuls représentants de leur discipline au sein de leur programme. Ils ne relèvent plus d'un chef de service formé dans la même discipline et les contacts avec un thérapeute senior sont plus rares. De plus, les ergothérapeutes et les physiothérapeutes doivent souvent superviser du personnel technique (assistants en ergothérapie et en physiothérapie). On s'attend aussi à ce que tous les thérapeutes participent à des activités d'appréciation de la qualité des services qu'ils rendent. Ce type de pratique plus autonome exige davantage d'habiletés d'analyse critique et de développement professionnel continu, ce que la maitrise permet davantage d'acquérir (Polotajko 1999; Salvatori 1998; University of Alberta 2000).

- Les ergothérapeutes travaillent aussi de plus en plus dans des milieux autres que le domaine de la santé. On peut les retrouver dans le domaine de l'éducation, en développement communautaire, dans le domaine de l'emploi (Strickland 1996). Ils ont donc besoin d'une vaste formation générale en plus de leurs solides connaissances en ergothérapie. Le fait de recevoir sa formation professionnelle au niveau de la maitrise permet d'acquérir une meilleure formation générale en arts et en sciences au niveau du baccalauréat (Warren \& Pierson 1994). 
Cette évolution des connaissances nécessaires à la pratique était déjà reconnue dans les faits par les divers programmes de baccalauréat en ergothérapie et en physiothérapie, puisque tous les programmes canadiens exigeaient déjà plus que les 120 crédits habituellement exigés dans un programme de baccalauréat. Certains programmes exigeaient même 60 ou 90 crédits universitaires préalables à l'admission à un programme de baccalauréat en ergothérapie ou en physiothérapie.

La seconde raison mentionnée est la reconnaissance professionnelle, dans un milieu de la santé où une proportion de plus en plus grande de professionnels sont formés au niveau de la maîtrise. Déjà en Ontario, plusieurs professionnels doivent posséder un doctorat (psychologie) ou une maîtrise professionnelle (audiologie et orthophonie) pour obtenir le droit de pratique ou pour pratiquer de façon autonome (service social) (Salvatori 1998). De plus, trois années d'études universitaires préalables sont exigées pour l'admission en médecine et à divers programmes en santé tels qu'optométrie et dentisterie.

À l'Université d'Ottawa, nous souscrivons aux arguments énoncés ci-dessus. Mais en plus, une autre motivation nous anime. Il nous apparait important que les étudiants franco-ontariens aient accès au même niveau d'éducation que leurs collègues anglophones et que la population franco-ontarienne ait accès à des soins dispensés par des professionnels ayant les mêmes compétences que la population anglophone. Ainsi, depuis septembre 2000, nous travaillons à définir nos nouveaux programmes de maitrise professionnelle en ergothérapie et en physiothérapie.

\section{Qu'est-ce qu'une maîtrise professionnelle?}

Selon le Conseil ontarien des études supérieures (OCGS/COES 1998) une maitrise professionnelle est un programme offert aux étudiants détenteurs d'un baccalauréat de diverses disciplines, qui propose un ensemble coordonné de cours pour préparer à l'exercice d'une profession ou à l'extension de la base de connais- 
sances nécessaires pour des professionnels en exercice. Un tel programme doit contenir certains éléments pour assurer le développement d'habiletés d'analyse et d'interprétation (analyse critique de la recherche). On distingue la maitrise professionnelle de la maitrise de recherche. Cette dernière est offerte à des étudiants qui ont un baccalauréat dans une discipline donnée et contient des cours avancés dans cette discipline, ainsi que le défi de la recherche intensive qui résulte habituellement en un mémoire de maitrise. Elle est la voie privilégiée pour préparer des études doctorales et une carrière en recherche.

Les maîtrises professionnelles d'accès à la pratique (formation de base) peuvent coexister avec des maitrises de recherche ou des maîtrises professionnelles visant une spécialisation pour les personnes qui possèdent un baccalauréat en ergothérapie ou en physiothérapie.

À l'Université d'Ottawa, nous envisageons que pour être admis à la maitrise professionnelle (formation de base), les étudiants devront avoir un baccalauréat dans un domaine de leur choix, incluant plusieurs cours préalables qui restent à être déterminés (des exemples de préalables possibles incluent : anatomie, psychologie, introduction aux méthodes statistiques et à la recherche). La formation à la profession comportera six trimestres d'études répartis sur deux ans, plutôt que les huit trimestres répartis sur quatre ans des programmes actuels de baccalauréat. Toutefois, ces cours seront tous des cours de la discipline, les cours de formation générale ayant été complétés avant l'admission au programme de maîtrise. Pour les étudiants de l'Ontario, cela signifiera six ans d'études universitaires pour devenir ergothérapeutes ou physiothérapeutes. Mais puisque ce changement survient en même temps que la réforme du secondaire (élimination de la $13^{\mathrm{e}}$ année), la formation totale ne durera qu'une année de plus que la formation actuelle au baccalauréat. De plus, plusieurs de nos étudiants actuels ont déjà un premier baccalauréat avant l'entrée dans les programmes d'ergothérapie ou de physiothérapie. Pour cette catégorie d'étudiants, la durée de la formation sera raccourcie de deux années pour n'être que de six ans, au lieu des huit qu'ils complètent actuellement. 
Le but des programmes de maitrise en ergothérapie et en physiothérapie sera de former des ergothérapeutes et des physiothérapeutes capables de porter un regard scientifique sur leur pratique, tout en possédant de solides habiletés cliniques et les qualités humaines nécessaires à la pratique centrée sur le client. Ces professionnels seront soucieux de leur développement professionnel continu, pourront remettre en question leur pratique, puiser, dans les résultats de recherche, les données probantes concernant leurs activités cliniques, et identifier des besoins de recherche là où ces données sont insuffisantes.

\section{L'enseignement dans un programme de maîtrise professionnelle}

Dans un programme de premier cycle, les étudiants acquièrent des connaissances générales ou spécialisées, mais aussi des habitudes de travail. Ils apprennent à rechercher des informations diverses dans des sources variées, à en faire l'analyse et la synthèse. Ainsi, les étudiants entrant dans un programme de maitrise, forts de cette expérience, seront plus autonomes et responsables dans leurs apprentissages, dès les premières heures de cours. Par exemple, ils sauront comment compléter les connaissances transmises par les professeurs par des lectures personnelles. Notre enseignement tiendra compte de ces caractéristiques et stimulera les étudiants à poursuivre des réflexions approfondies sur les sujets de leur choix.

De plus, ces habiletés génériques acquises par les étudiants au premier cycle nous permettront d'augmenter l'accent mis sur la recherche au sein du programme. Outre le projet de recherche qui permettra aux étudiants de démontrer leur compréhension du processus de la recherche, l'analyse de la recherche actuelle en réadaptation sera intégrée à l'enseignement de chacun des sujets du programme. À l'intérieur de chaque cours, les étudiants pourront avoir à faire une analyse critique d'articles de recherche sur une approche théorique ou sur une méthode clinique donnée. 
Si la recherche est peu développée sur un sujet, ils seront appelés à identifier des moyens qui permettraient d'obtenir davantage de données scientifiques sur ce sujet. Les étudiants seront aussi appelés à utiliser leur esprit d'analyse pour discuter des enjeux professionnels et éthiques rencontrés en réadaptation.

\section{Signification pour la pratique de l'ergothérapie et de la physiothérapie}

Le fait d'avoir davantage d'ergothérapeutes et de physiothérapeutes mieux formés à la recherche ne peut que renforcer nos professions, dans un contexte où l'on doit de plus en plus démontrer que nos interventions sont fondées sur des faits scientifiques. La formation de niveau maitrise peut amener une meilleure reconnaissance de la profession et par conséquent, de plus grandes possibilités pour les ergothérapeutes et les physiothérapeutes d'occuper divers rôles de consultants et de participer à la définition des politiques en matière de santé et de bien-être. Le public ne pourra que bénéficier de l'élargissement des perspectives, si les connaissances acquises dans le domaine de la réadaptation sont davantage intégrées aux politiques de santé.

Il demeure cependant que, tout comme les orthophonistes et les audiologistes qui ont une formation de niveau maitrise, les ergothérapeutes et les physiothérapeutes formés à la maitrise continueront à faire des interventions cliniques incluant l'évaluation et la conduite de la thérapie auprès des clients. Comme c'est déjà le cas actuellement, certains actes thérapeutiques peuvent être confiés à du personnel technique (assistants en ergothérapie et en physiothérapie), mais les éléments plus complexes des thérapies, ainsi que toutes les décisions concernant celles-ci, demeureront la responsabilité des professionnels : les ergothérapeutes et les physiothérapeutes. Si les professionnels formés à la maîtrise ont de meilleures habiletés pour analyser les résultats 
de recherche et pour les intégrer plus rapidement à leur pratique, ils pourront en faire bénéficier leurs collègues et leurs clients.

Par ailleurs, les nouveaux finissants formés à la maitrise et les professionnels actuellement en exercice formés au baccalauréat posséderont des connaissances complémentaires. En effet, il faut s'assurer de maintenir la valeur des connaissances acquises par l'expérience. Les professeurs des programmes d'ergothérapie et de physiothérapie reconnaissant la richesse de cette expérience, s'assureront de la mettre à profit dans les programmes de maîtrise, en continuant à faire appel à des thérapeutes d'expérience pour diverses parties de la formation théorique et clinique des étudiants.

Les associations professionnelles reconnaissent aussi les connaissances acquises par l'expérience des professionnels actuellement en exercice. Elles ne parlent pas, pour le moment, d'exiger une formation de mise à niveau. Et si le passé est garant de l'avenir, historiquement, de tels changements se sont faits de façon progressive et il y a eu reconnaissance des diplômes des professionnels en exercice (par ex., lorsque la formation est passée du diplôme au baccalauréat). Les professionnels actuellement en exercice qui souhaitent obtenir une maitrise auront plusieurs possibilités, offertes par diverses universités : maitrise de recherche en réadaptation, maîtrise professionnelle de spécialisation en réadaptation, maîtrise dans une discipline connexe (sciences neurologiques, épidémiologie, éducation, administration, etc. ) pour aller chercher des connaissances complémentaires.

\section{Étapes à suivre pour la modification du programme}

Des groupes de travail comprenant des cliniciens et des professeurs ont été formés dans chacune des disciplines pour aider à définir les nouveaux programmes de maitrise professionnelle. ${ }^{3}$ Nous avons déjà défini les buts des nouveaux programmes. Nous avançons dans l'élaboration des contenus à couvrir, la définition des objectifs spécifiques d'apprentissage et l'élaboration du programme de cours. 
L'ensemble des cours, le travail de recherche et un total d'environ 1000 heures de formation clinique composeront chacun des nouveaux programmes. Nous travaillons aussi à définir les stratégies à mettre en place pour assurer les ressources nécessaires pour offrir ces nouveaux programmes. Il faudra de plus obtenir l'approbation des différentes instances de l'Université d'Ottawa et du Conseil ontarien des études supérieures. Une fois l'approbation obtenue, un travail de communication suivra pour informer adéquatement les candidats et les diverses personnes qui travaillent à la promotion des programmes et au processus d'admission. Les changements pourraient entrer en vigueur entre 2004 et 2008.

Soulignons que pour septembre 2002 et 2003 (année de la double cohorte dans les écoles secondaires de l'Ontario), nos programmes d'ergothérapie et de physiothérapie demeurent accessibles aux étudiants du secondaire qui remplissent les conditions d'admission.

\section{Conclusion}

Cet article voulait faire connaitre les transformations qui ont lieu dans les programmes d'ergothérapie et de physiothérapie. En collaboration avec de nombreux cliniciens consultants intéressés à participer à l'élaboration de ces programmes, les professeurs en réadaptation à l'Université d'Ottawa veulent relever le défi lancé par les organisations professionnelles pour une formation mieux adaptée à l'évolution des connaissances, des rôles et des programmes de soins de santé.

\section{Références}

AMERICAN OCCUPATIONAL THERAPISTS ASSOCIATION (2002). ACOTE (Accreditation Council for Occupational Therapy Education) Sets Timeline for Postbaccalaureate Degree Programs (Reprinted from OT Week, 13(33)). Extrait du site Web http://www. aota. org/nonmembers/ area13/links/link16. asp, le 15 février 2002. 
ASSOCIATION CANADIENNE DES ERGOTHÉRAPEUTES (28 novembre 2001). Feuillet d'information sur les changements apportés aux exigences en matière d'éducation. Extrait du site Web http://www. caot. ca/index. cfm?URLclick=78, le 7 février 2002.

ASSOCIATION CANADIENNE DE PHYSIOTHÉRAPIE (2001). Vision for Entry-Level Education for Physiotherapists in Canada.

COMMISSION ON ACCREDITATION IN PHYSICAL THERAPY EDUCATION (1997). "Commission Adopts New Evaluative Criteria for PT Programs ". Accreditation Update, septembre, 2.

ONTARIO COUNCIL ON GRADUATE STUDIES (1998). OCGS Statutes, By-laws, Procedures and Guidelines.

LAW, Mary, BAUM, Carolyn (1998). «Evidence-Based Occupational Therapy - La pratique de l'ergothérapie fondée sur l'évidence scientifique ». Revue canadienne d'ergothérapie / Canadian Journal of Occupational Therapy, 65:3, 131-135.

POLOTAJKO, Helene, MILLER-POLGAR, Jan, COOKE, Joanne (1999). " Les programmes de maitrise en ergothérapie : Preuve de vision ou reflet de maturité de la profession? " Actualités ergothérapiques, 1:1, janvier, 7.

SALVATORI, Penny (1998). "Hot Issues: Masters Entry Level Education in Occupationnal Therapy in Canada ", The Link, 49, août.

STRICKLAND, Anne (1996). "Trends and transitions ", National, mai, 4-5.

UNIVERSITY OF ALBERTA, DEPARTMENT OF PHYSICAL THERAPY (2000). «Rationale for developing a proposal for a Master's Entry Level PT Program at U of A », On the Move... Physical Therapy curriculum innovations, 1:1, septembre, 1-2.

WARREN, Stacey C. , PIERSON, Frank M. (1994). "Comparison of characteristics and attitudes of entry level bachelor's ans master's degree students in physical therapy ", Physical Therapy, 74:4, avril,333-348.

\section{Notes}

1. Veuillez adresser toute correspondance au sujet de cet article à Jacinthe Savard, téléphone : (613) 562-5800, poste 8393, télécopieur : (613) 562-5428, courriel : jsavard@uottawa.ca.

2. Ergothérapie se traduit en anglais par Occupational Therapy.

3. Nous voulons remercier les personnes qui travaillent à l'élaboration des nouveaux programmes de maîtrise professionnelle en ergothérapie et en physiothérapie.

Membres du comité ad hoc sur la maitrise professionnelle en ergothérapie : Mary Egan, erg, Ph.D., Jean-Pascal Beaudoin, erg, Lara Haddad, erg, Diana MacIntosh, erg, Hélène Martin, erg, Danielle Massicotte, erg, DES(adm. publique), Catherine Vallée, erg. MSc.

Membres du comité ad hoc sur la maitrise professionnelle en physiothérapie :Dawn Burnett, pht, MA, Wilma Jelley, pht, Lucie Pelland, pht, PhD.,Lucie Poulin, erg, MA., Tina Alverez-Wiest, pht, Gail Bowes, pht, Jean-Jacques Goldin, pht, Lorraine Hendry, pht, Ali Muir, pht.. 